\title{
Alterations in protein secretion caused by metabolic engineering of glycosylation pathways in fungi
}

\author{
Joanna S. Kruszewska®, Urszula Perlińska-Lenart, Wioletta Górka-Nieć, \\ Jacek Orłowski, Patrycja Zembek and Grażyna Palamarczyk \\ Institute of Biochemistry and Biophysics, Polish Academy of Sciences, Warszawa, Poland
}

Received: 07 July, 2008; revised: 28 August, 2008; accepted: 15 September, 2008

available on-line: 17 September, 2008

\begin{abstract}
Due to its natural properties, Trichoderma reesei is commonly used in industry-scale production of secretory proteins. Since almost all secreted proteins are O-glycosylated, modulation of the activity of enzymes of the O-glycosylation pathway are likely to affect protein production and secretion or change the glycosylation pattern of the secreted proteins, altering their stability and biological activity. Understanding how the activation of different components of the O-glycosylation pathway influences the glycosylation pattern of proteins and their production and secretion could help in elucidating the mechanism of the regulation of these processes and should facilitate creation of engineered microorganisms producing high amounts of useful proteins. In this review we focus on data concerning Trichoderma, but also present some background information allowing comparison with other fungal species.
\end{abstract}

Keywords: glycosylation, protein secretion, Trichoderma

\section{INTRODUCTION}

Trichoderma species play an important role in the biotechnological industry where their protein synthesis and secretory capabilities are widely exploited for protein production. Hence, stimulation of their secretory capacity is of considerable commercial interest and novel potential stimulants of protein production and secretion are highly desirable. Some species of Trichoderma secrete up to $40 \mathrm{~g}$ of protein per liter of culture (Durand et al., 1988), however, the yield of heterologously expressed proteins is not so impressive.

In $T$. reesei the majority of secretory proteins (Table 1) are highly glycosylated with both $\mathrm{N}$ - and O-linked glycans (Table 2) (Palamarczyk et al., 1998). In this paper we present the current understanding of the interdependencies between protein glycosylation and their production, secretion, and activity. In particular, we describe the influence of changes in the activity of the O-glycosylation pathway on protein production by Trichoderma.

\section{TRICHODERMA SECRETORY PROTEINS ARE HIGHLY GLYCOSYLATED}

It was discovered by our group that protein O-glycosylation in Trichoderma occurred in a similar way as in the yeast Saccharomyces cerevisiae (Fig. 1) (Kruszewska et al., 1989). The first mannosyl residue transferred by protein $\mathrm{O}$-mannosyltransferases onto the $\mathrm{OH}$-group of serine (Ser) or threonine (Thr) originates from dolichyl phosphate mannose (DPM) and then, for the elongation of the sugar chain, GDPmannose is used. The significance of this process becomes obvious when we consider the structure of proteins secreted by Trichoderma. Cellobiohydro-

${ }^{\square}$ Corresponding author: Institute of Biochemistry and Biophysics, Polish Academy of Sciences, Adolfa Pawińskiego 5a, 02-106 Warszawa, Poland; phone (48 22) 592 1209; fax: (48 22) 658 4636; e-mail jsk@ibb.waw.pl

Abbreviations: GDP-mannose, guanosine diphosphate mannose; Dol-P, dolichyl phosphate; DolPMan or (DPM), dolichyl phosphate mannose; DolPGlc, dolichyl phosphate glucose; GlcNAc, $N$-acetylglucosamine; UDP-N-acetylglucosamine, uridine diphosphate $\mathrm{N}$-acetylglucosamine. 
lase I (CBHI), the main hydrolytic enzyme secreted by this fungus, is composed of a catalytic and a cellulose binding domain, connected by an O-glycosylated linker (Fagerstam et al., 1984). A major function of the O-glycosylation of the linker is to maintain a fixed distance between the catalytic and binding domains, as has been shown for glucoamylase from Aspergillus niger (Williamson et al., 1992).

In the high-secreting Trichoderma strain ALKO 2877 the glycosylated forms of the linker contain from 14 to 26 hexoses (Harrison et al., 1998). All threonines in the linker are glycosylated, with at least one and up to three mannoses per site. In CBHI secreted by Trichoderma RutC-30 strain the linker is extensively glycosylated at threonine and serine residues with di- and tri-saccharides, and in addition some phosphorylated di-saccharides are also found (Hu et al., 2001).

The proteins secreted by Trichoderma are also N-glycosylated. The N-glycosylation process is conserved in Eukaryotes and requires dolichyl phosphate as oligosaccharide carrier [DolPP$(\mathrm{GlcNAc})_{2} \mathrm{Man}_{9} \mathrm{Glc}_{3}$ ] synthesized step by step using UDP- $N$-acetylglucosamine, GDP-mannose, DolPMan and DolPGlc (Helenius \& Aebi, 2001; Wildt \& Gerngross, 2005). The whole oligosaccharide is transferred by oligosaccharyl transferase to an asparagine located in the consensus sequence (-Asn-X-Ser/Thr-) in the protein (Yan \& Lennarz, 2005; Lennarz, 2007).

$\mathrm{CBHI}$ has four $\mathrm{N}$-glycosylation sites, all in the catalytic domain (Swiss-Prot P62695). The structure of the N-linked saccharides depends on the fungal strain and conditions of cultivation (Klarskov et al., 1997; Maras et al., 1997; Pakula et al., 2000; Garcia et al., 2001; Hui et al., 2001; 2002; Harrison et al., 2002; Stals et al., 2004). Cultivation in minimal media, when the medium acidifies, results in fully glycosylated and phosphorylated proteins, while rich media stimulate the activity of endoglucosidase $\mathrm{H}$

Table 1. Main glycoproteins produced by Trichoderma

\begin{tabular}{lll}
\hline Enzyme & EC number & NCBI or Reference \\
\hline${ }^{*}$ Cellobiohydrolase I (CBHI) & EC 3.2.1.91 & Shoemaker et al., 1983 \\
${ }^{*}$ Cellobiohydrolase II (CBHII) & EC 3.2.1.91 & AAA34210 \\
${ }^{*}$ Endoglucanase I (EGI) & EC 3.2.1.4 & AAA34212 \\
${ }^{*}$ Endoglucanase II (EGII) & EC 3.2.1.4 & ABA64553 \\
Endoglucanase III (EGIII) & EC 3.2.1.4 & AAA34213 \\
Endoglucanase IV (EGIV) & EC 3.2.1.4 & CAA71999 \\
Endoglucanase V (EGV) & EC 3.2.1.4 & P43317 \\
${ }^{*} \alpha$-Galactosidase (melibiase) & EC 3.2.1.22 & Savel'ev et al., 1997 \\
${ }^{* \beta-G a l a c t o s i d a s e ~}$ & EC 3.2.1.23 & Gamauf et al., 2007 \\
B-Glucosidase & EC 3.2.1.21 & BAA74959 \\
Chitinase 46 & EC 3.2.1.14 & BAD44715 \\
Chitinases CHI 1-18 from family 18 & EC 3.2.1.14 & Seidl et al., 2005 \\
${ }^{*}$ Acetylxylan esterase & EC 3.1.1.6 & CAA93247 \\
\hline
\end{tabular}

*structure of carbohydrates characterized by Klarskov et al. (1997); Maras et al. (1997); Harrison et al. (1998; 2002); Hui et al. (2002).
(Endo $\mathrm{H}$ ), mannosidases and phosphatases, thus modifying the pattern of the N-linked oligosaccharides.

CBHI from T. reesei QM9414 cultivated with lactose-cellobiose (9: 1) was reported to have only single $\mathrm{N}$-acetylglucosamine (GlcNAc) residues linked to asparagines in positions 45, 270 and 384 in the catalytic domain (Klarskov et al., 1997). On the other hand, the RutC-30 strain may also secret CBHI with a high-mannose glycan (predominantly $\mathrm{GlCNAc}_{2} \mathrm{Man}_{8}$ ) with outer-branch phosphodiester-mannose linkages attached to Asn270 and a single GlcNAc at Asn45 and Asn384 (Maras et al., 1997; Hu et al., 2001; Stals et al., 2004). It has also been reported that $T$. reesei secretes multiple forms of CBHI, and in one isolation 14 different glycoforms were found (Garcia et al., 2001). The major isoform contained only one GlcNAc in the catalytic domain, presumably N-linked, and one mannose, most probably O-linked to serine/threonine at a separate site. Except for a small fraction of the enzyme containing GlcNAc${ }_{2} \mathrm{Man}_{5}$ +1-2 Man, the majority of the protein molecules had negatively charged phosphate-containing N-glycans. All glycoforms contained at least one O-linked mannose residue.

Intensive $\mathrm{O}-$ and N-glycosylation and different glycoforms were also shown for other Trichoderma secretory proteins such as cellobiohydrolase II (CBHII) and endoglucanases (EGI, II) (Hui et al., 2002). Glycosylation of these enzymes accounted for $12-24 \%$ of their molecular mass. Both CBHII and endoglucanases contained high-mannose forms of glycans as well as a single GlcNAc attached to asparagine.

In spite of the intensive study of the N-glycoforms of proteins secreted by Trichoderma, the biological significance of $\mathrm{N}$-linked carbohydrates in the catalytic domain is not known. It is known, however, that the proper course of N-glycosylation is tightly connected with protein folding and crucial for the recognition of misfolded glycoproteins which are then retained in the endoplasmic reticulum (Parodi, 2000). Accumulation of misfolded proteins in the endoplasmic reticulum causes secretion stress leading to transcriptional down regulation of genes encoding secreted proteins in $T$. reesei and A. niger (Pakula et al., 2003; AlSheikl et al., 2004).

Prevention of correct protein folding in Trichoderma by incubation with dithiothreitol leads to enhanced expression of components of the UPR (unfolded protein response) pathway such as foldases and chaperones (Pakula et al., 2003). A global transcrip- 
Table 2. O- and N-linked carbohydrates found in Trichoderma glycoproteins

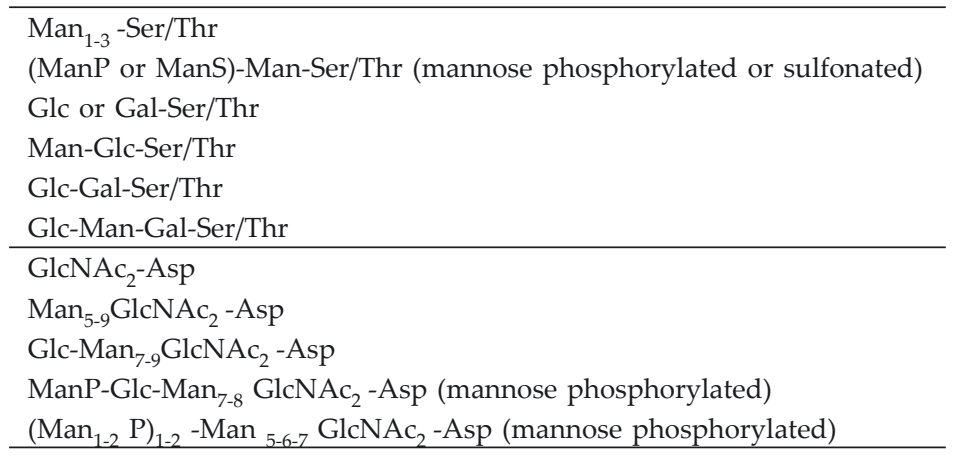

Man, mannose; Glc, glucose; Gal, galactose; GlcNAc, N-acetylglucosamine; These carbohydrates structures are presented by Salovuori et al. (1987); Savel'ev et al. (1997); De Bruyn et al. (1997); Maras et al. (1997); Hui et al. (2001); Stals et al. (2004); Goto (2007).

tional analysis of the stress response in $A$. niger has revealed up-regulation of chaperones, foldases, glycosylation enzymes, vesicle transport proteins, and ER-associated proteases (Guillemette et al., 2007). These results indicate possible targets for manipulation in strain improvement strategies. From the biotechnological point of view the most important is, however, that dysfunction of glycosylation results in accumulation of proteins in the endoplasmic reticulum and a drastic down regulation of the genes encoding secreted proteins (Pakula et al., 2003; Guillemette et al., 2007).

\section{IMPORTANCE OF THE GLYCOSYLATION PATTERN}

The correct glycosylation pattern is particularly important with regard to therapeutic or enzymatic proteins (Hollister et al., 2002; Ahn et al., 2008; Jenkins et al., 2008; Ko et al., 2008; Spiriti et al., 2008). Altered glycosylation may affect the stability and half-life of the protein, and change its activity or affinity towards some substrates. Hyperglycosylation of CBHII expressed in S. cerevisiae reduced its affinity towards crystalline cellulose. The poor binding to cellulose causes the heterologously expressed CBHII to aggregate more readily than the native enzyme secreted by Trichoderma (Penttila et al., 1988). Similar results were obtained when Trichoderma CBHI was expressed in A. awamori (Jeoh et al., 2008). The recombinant enzyme contained six-fold more of $\mathrm{N}$ linked glycan than the enzyme secreted by Trichoderma. That hyperglycosylation resulted in a decreased enzymatic activity and lower affinity towards cellulose, while cleavage of the $\mathrm{N}$-glycans by $\mathrm{N}$-glucosidase (PNGaseF) improved both parameters. On the other hand, hyper-N-glycosylated endoglucanase
II (EGII) produced in yeast revealed a wider $\mathrm{pH}$-range of activity and higher thermal stability compared to the native enzyme (Qin et al., 2008). The two enzymes differed in their activity toward various substrates and the recombinant one had a higher activity against Avicel cellulose. Endoglucosidase $\mathrm{H}$ treatment of the hyperglycosylated EGII restored about $88 \%$ of its original activity. A decreased O-glycosylation of glucoamylase I from A. awamori expressed in an S. cerevisiae pmt1 disruptant resulted in a $70 \%$ decrease of the activity towards raw starch compared to the enzyme expressed in a wild type $S$. cerevisiae strain (Goto et al., 1999). In addition, the stability of the under-glycosylated glucoamylase I toward extreme $\mathrm{pH}$ and high temperature was reduced.

In general, under-glycosylated proteins could be recognized by the protein quality control system and the non-native proteins could be directed to the

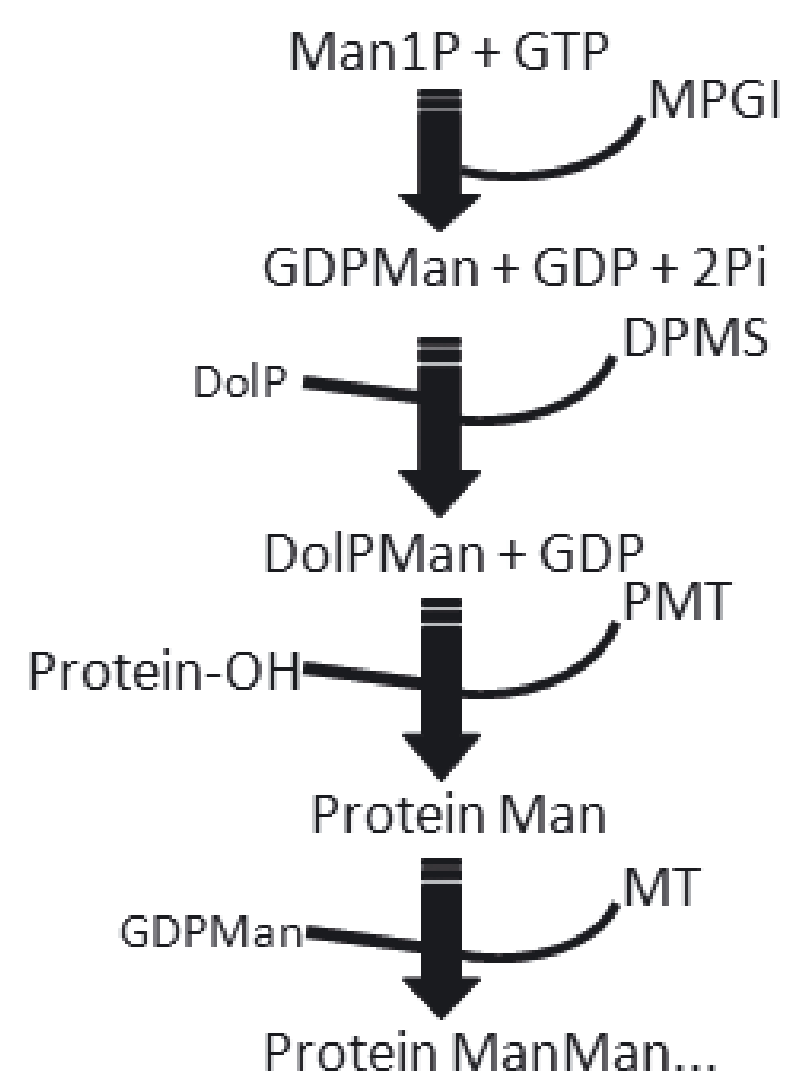

Figure 1. O-glycosylation pathway in fungi. GDPMan, guanosine diphosphate mannose; Man1P, mannose-1-phosphate; DolP, dolichyl phosphate; Man, mannosyl residue; MPGI, guanylyltransferase; DPMS, dolichyl phosphate mannose synthase; PMT, protein O-mannosyltransferase; MT, mannosyltransferase. 
retrograde transport and degraded by the ER-associated degradation (ERAD) pathway (Harty et al., 2001; Ellgaard \& Helenius, 2003; Nakatsukasa et al., 2004). It was also shown that substitutive O-glycosylation of proteins mutated in the $\mathrm{N}$-glycosylation sites restored their solubility and they were partly secreted out of the cell through the normal secretory pathway (Nakatsukasa et al., 2004).

There is no doubt that the glycosylation status of enzymatic proteins influences their important parameters, and understanding these relationships allows a proper strategy to be adopted for the enzymes' production. One may choose optimal cultivation conditions or use a different host for heterologous expression of the protein of interest. Also the glycosylation pathways may be engineered to influence directly the glycosylation pattern of produced proteins (Choi et al., 2003; Wildt \& Gerngross, 2005).

\section{DISTURBED GLYCOSYLATION MAY CREATE A BARRIER FOR PROTEIN PRODUCTION AND SECRETION}

A number of studies underline the important role of O-glycosylation of secretory proteins for their production and secretion (Kubicek et al., 1987; Kruszewska et al., 1990; 1999; Agaphonov et al., 2001; 2005; Perlińska-Lenart et al., 2006a). We have suggested previously (Kruszewska et al., 1990) that dolichyl phosphate mannose synthase (DPM synthase; EC 2.4.1.8.80) plays a key role in T. reesei O-glycosylation. In Trichoderma, like in the human, DPM synthase forms an enzymatic complex of three different proteins DPMI, DPMII and DPMIII. In human the Dpm1 protein is the catalytic subunit attached to the endoplasmic reticulum via the Dpm3 protein and regulated by Dpm2 (Maeda et al., 1998, 2000; Ashida et al., 2006). The DPM synthase activity is 10 -fold higher in the presence of Dpm2 protein, indicating that this protein plays an important role in the enzymatic reaction, however, it is not essential for survival. In the yeast $S$. cerevisiae DPM synthase is encoded only by the DPM1 gene and the Dpm1 protein itself has an enzymatic activity. The protein has a C-terminal transmembrane domain which is not present in the DPM1 protein from the human class of DPM synthases (Colussi et al., 1997), however, addition of the yeast transmembrane domain to Trichoderma DPMI was not sufficient to rescue the S. cerevisiae $\Delta d p m 1$ mutant (Kruszewska et al., 2000).

Based on the finding that Dpm1 protein from S. cerevisiae is enzymaticaly active, the DPM synthase activity was elevated in Trichoderma by overexpression of the yeast DPM1 gene. The transform- ants had an over twice higher activity of the enzyme and, more importantly, secreted up to seven-fold higher amount of proteins (Kruszewska et al., 1999). Moreover, the proteins, although secreted in a huge amount, were glycosylated to the same extent as in the control strain.

A detailed study of the Trichoderma DPM1transformed strains revealed significant changes in the structure of their cell wall. The secretion process is very well documented in fungi and some potential barriers for protein secretion are recognized (Conesa et al., 2001). The cell wall is an evident barrier for protein secretion. In an $A$. nidulans strain expressing the DPM1 gene from $S$. cerevisiae, protein production was elevated similarly as it was in the Trichoderma DPM1 transformants, but since the cell wall of the A. nidulans transformant was not altered, the secreted proteins were accumulated in the periplasmic space (PerlińskaLenart et al., 2005). On the other hand, removal of the cell wall of Trichoderma did not change protein production and secretion in the wild type strain, however, for a strain with an increased potential to produce and modify proteins, such as the DPM1-expressing strain, the cell wall was indeed shown to limit the protein secretion (PerlińskaLenart et al., 2006b). There are examples of how changes found in the cell wall structure could influence the process of fermentation. Disruption of the chitin synthase gene chsB in Aspergillus altered growth and morphology of the fungus manifested as hyperbranching and sensitivity to Calcofluor White (Müller et al., 2002). The hyperbranching of the mycelia decreased their clumping which in turn facilitated oxygen uptake by the cells and improved the flow of substrates and products. Intensive branching of mycelia was also observed in A. fumigatus carrying disruption of the AGS1 gene encoding $\alpha-1-3$-glucan synthase and in consequence characterised by a reduced content of $\alpha-1$ 3-glucan (Beauvais et al., 2005). Carbohydrate polymers are the main components of the fungal cell wall, however, mannosylated proteins make up about $40 \%$ of its composition in the yeast (Kollar et al., 1997; Schmidt et al., 2005; Klis et al., 2006). It was suggested that defects in protein glycosylation could impair cell wall integrity by influencing the folding and activity of proteins catalysing synthesis of cell wall components (Chavan et al., 2003). It was proposed that a mutation in STT3, encoding an essential subunit of the oligosaccharyl transferase complex catalysing transfer of the oligosaccharide chain from dolichyl phosphate (DolP) to protein, led to an insufficient synthesis of the glucan primer serving as a building block for $\beta-1$ 6 -glucan biosynthesis. Moreover, it was suggested that $\mathrm{N}$-linked sugars of certain secretory proteins 
or the cell wall mannoproteins themselves could serve as the initial acceptor of glucosyl residues to generate the $\beta-1-6$-glucan chain (Shahinian et al., 1998).

\section{INCREASED ACTIVITY OF GUANYLYLTRANSFERASE OR cis- PRENYLTRANSFERASES ENHANCES GLYCOSYLATION OF SECRETED PROTEINS}

DPM synthase, a key enzyme of the O-glycosylation pathway, uses GDP-mannose and dolichyl phosphate as substrates for production of dolichyl phosphate mannose (DPM). Shortage of the substrates decreases the production of DPM and limits protein O-glycosylation as well as N-glycosylation and the synthesis of the GPI anchor. Overexpression of the mpg1 gene coding for guanylyltransferase, the enzyme synthesizing GDP-mannose, increased the cellular level of GDP-mannose in T. reesei (Zakrzewska et al., 2003). In the mpg1-overexpressing strains the increased activity of DPM synthase was accompanied by an increased $\operatorname{dpm} 1$ transcript level and a higher concentration of DPMI protein. Since a simple increase in $d p m 1$ mRNA and DPMI protein does not ensure an increase in DPM synthase activity, as demonstrated for Trichoderma transformed with its own dpm 1 gene (Zakrzewska et al., 2003), one might speculate that the overexpression of $m p g 1$ resulting in an increased dpm1 mRNA level also increases expression of the other subunits of DPM synthase, i.e., DPMII and DPMIII proteins in T. reesei. The higher level of GDP-mannose in the cells also activated mannosyltransferases elongating O-linked carbohydrate chains. In turn, the elevated activity of the mannosyltransferases resulted in longer O-linked carbohydrates bound to the secreted proteins. On the other hand, we also observed more intensive $\mathrm{N}$-glycosylation of the proteins as a result of the increased pools of DPM and GDP-mannose in the cell. The mpg1-overexpressing strains showed no changes in the amount of proteins secreted, however, this modification of Trichoderma could still be beneficial for biotechnology because the secreted cellulases were by up to $50 \%$ more active than those secreted by the wild type strain (our unpublished data). The lack of changes in the amount of secreted proteins could have resulted from the unchanged cell wall of the transformants.

The influence of overproduction of GDP-mannose on the formation of fungal cell wall appears to be species-specific. Overexpression of the guanylyltransferase PSA1 (MPG1) gene in the yeast Kluyveromyces lactis wild type strain JA6 resulted in a higher resistance to Hygromycin B and SDS and enabled the cells to grow on medium with $50 \mu \mathrm{g} \mathrm{ml}^{-1}$ Cal- cofluor White, often used as a diagnostic tool for detection of cell wall changes (Uccelletti et al., 2005). The transformation of $K$. lactis with the PSA1 gene increased expression and secretion of human serum albumin from a cDNA construct three-fold compared with that in the JA6 strain; this was not due to an enhanced transcription of the human cDNA. Similarly, expression of glucoamylase from the salttolerant yeast Arxula adeninivorans in the modified $K$. lactis strain gave a significantly more active enzyme than the one secreted by the control strain.

Here, however, the authors reported on the enzyme's total activity only, so it is not known whether this effect was due simply to an increased secretion of the protein, its higher specific activity that could have been caused by an altered glycosylation, or both.

Attempts to elucidated the reasons of the enhanced production of heterologous proteins by $K$. lactis cells overexpressing the PSA1 (MPG1) gene revealed a slight increase of its cell wall porosity (Uccelletti et al., 2005). Consequently, the authors suggested that the enhanced secretion might have been connected not with an enhanced glycosylation potential of the $K$. lactis strain overexpressing the PSA1 gene, but rather with its modified cell wall. After all, the highly secreted human serum albumin has no $\mathrm{N}$-glycosylation sites and probably was also not O-glycosylated. On the other hand, shortage of GDP-mannose in a Hansenula polymorpha conditional mutant partially blocked in GDP-mannose production influenced O-glycosylation, and this defect in glycosylation decreased the secretion of a model Oglycosylated protein, chitinase, which was poorly glycosylated (Agaphonov et al., 2001). A comparison of the glycosylation and secretion of the N-glycosylated invertase with the above-mentioned effect on the O-glycosylated chitinase revealed differences in the effect of these two types of glycosylation on protein secretion. Invertase, a heavily $\mathrm{N}$-glycosylated enzyme due to the presence of four $\mathrm{N}$-glycosylation sites, was secreted in a larger amount by the mutant than by the wild type strain, despite being totally non-glycosylated. Similarly, expression of the N-glycosylated human urinary type plasminogen activator (u-PA) in the Hansenula mutant resulted in the secretion of an unglycosylated, active protein, but only by the mutant strain, which could be due to its more permeable cell wall.

On the other hand, it was shown that expression of a potentially highly glycosylated protein itself activated enzymes of the O-glycosylation pathway (Górka-Nieć et al., 2007). Expression of Trichoderma cellobiohydrolase II in S. cerevisiae resulted in a significant activation of protein $\mathrm{O}$-mannosyltranferases, mannosyltransferases elongating O-linked carbohydrates, and cis-prenyltransferase. 
Similarly to the shortage of GDP-mannose, also limitations in dolichyl phosphate production caused by low activity of cis-prenyltransferase (Shenk et al., 2001) could influence protein glycosylation and secretion. Dolichyl phosphate serves as a carrier of carbohydrate residues in $\mathrm{O}$ - and $\mathrm{N}$-glycosylation by providing the first mannosyl residue for the direct transfer to the $-\mathrm{OH}$ group of serine and threonine in protein O-glycosylation, and by taking part in the synthesis of lipid-linked oligosaccharide in the N-glycosylation process. To overcome the possible shortage of dolichyl phosphate for the glycosylation of secretory proteins in Trichoderma, the yeast RER2 gene was overexpressed in this fungus. RER2 encodes cis-prenyltransferase, a key enzyme in dolichol synthesis, the first of the polyprenol branch of the mevalonate pathway (Sato et al., 1999; 2001). The enzyme catalyzes the elongation of polyprenol chain by sequential addition of isopentenyl diphosphate (IPP) to farnesyl diphosphate (FPP) (Daleo et al., 1977; Adair \& Cafmeyer, 1987; Szkopińska et al., 1996).

Overexpression of the yeast RER2 gene in $T$. reesei caused no significant changes in protein secretion, however, the secreted proteins were more heavily $\mathrm{O}-$ and N-glycosylated (PerlińskaLenart et al., 2006a). The higher N-glycosylated proteins were secreted later during cultivation than O-glycosylated, in agreement with the concept of the controlling role of O-glycosylation in the N-glycosylation process (Ecker et al., 2003). At the same time, activation of the first two reactions in the formation of lipid-linked oligosaccharide during N-glycosylation was observed. The first steps of N-glycosylation require UDP- $N$-acetylglucosamine also used in the synthesis of chitin, which was temporarily decreased in the cell wall of the RER2- transformed Trichoderma strains.

Mutation in the RER2 gene in S. cerevisiae caused accumulation of ER membranes and their extreme elongation and, simultaneously, led to a ring-like structure of Golgi membranes (Sato et al., 1999); most importantly, it caused mislocalization of some of the ER proteins engaged in protein trafficking. All these changes point to a tight interdependence between Rer2p activity and the state of the cellular structures taking part in protein glycosylation and secretion. A limited activity of Rer2p (Belgareh-Touze et al., 2003; Shridas et al., 2003) caused underglycosylation of caboxypeptidase Y, a model N-glycosylated protein, however, the lack of Rer2p did not affect the plasma membrane Gas1 protein, which carries N- and O-linked sugars and, in addition, requires a glycosylphosphatidyl inositol (GPI) anchor. On the other hand, the O-glycosylated Hsp150 protein was found to be underglycosylated in cells when RER2 expression was inhibited (Davydenko et al., 2004).

\section{DISTURBED ACTIVITY OF PROTEIN $O$-MANNOSYLTRANSFERASES COULD ENHANCE SECRETION OF N-GLYCOSYLATED PROTEINS AND DECREASE SECRETION OF O- GLYCOSYLATED ONES}

Protein O-mannosyltransferases, enzymes directly transferring the mannosyl residue from dolichyl phosphate mannose to the -OH group of serine or threonine are represented in S. cerevisiae by seven Pmt proteins. This suggests an essential role of the O-glycosylation process for the survival of fungal cells. The yeast Pmt proteins are classified in three subfamilies PMT1, PMT2 and PMT4 (Gentzsch \& Tanner, 1996; 1997). Members of the PMT1 and PMT2 subfamilies, Pmt1p and Pmt5p, and Pmt2p, Pmt3p and Pmt6, respectively, form enzymatically active heterodimers such as Pmt1-Pmt2 and Pmt3Pmt5 (Girrbach \& Strahl, 2003). Deletion of a PMT gene encoding a protein from either of these groups results in the formation of less active complexes such as Pmt1-Pmt3 or Pmt2-Pmt5. The PMT4 family has one member only, Pmt4 p, and this protein forms an active homodimer. Moreover, protein O-mannosyltransferases are substrate-specific (Gentzsch \& Tanner, 1997).

Up to now, there are no data concerning the influence of an increased activity of protein $\mathrm{O}$-mannosyltransferases on protein production, glycosylation and secretion or on the assembly of the cell wall. Only the effects of deletion of the genes encoding protein O-mannosyltransferases have been studied. In $A$. nidulans, three Pmt proteins have been identified and it was shown that a lack of an individual Pmt protein resulted in cell wall damage, swollen hyphae, hyperbranching, reduced or no conidiation, an increased number of nuclei and the presence of non-O-glycosylated proteins (Goto, 2007). Disruption of the pmtA gene in $A$. awamori did not change the amount of secreted glucoamylase I, however, it caused a significant decrease of its O-glycosylation (59.4\%) but not N-glycosylation (Oka et al., 2005). Moreover, the authors also observed an alteration of the O-linked oligosaccharide profiles. The underO-glycosylated glucoamylase I had a lower specific activity toward soluble starch.

Disruption of the pmtA gene in A. awamori or $A$. nidulans resulted in abnormal cell morphology and alteration in carbohydrate composition of their cell wall but, nonetheless, the secretion of proteins by the mutants was not altered. Those results showed that weakening of the cell wall structure was not enough to stimulate protein production 
and secretion when O-glycosylation abilities were limited. In wild type $T$. reesei removing of the cell wall did not result in increased protein production and secretion, while DPM1-transformed Trichoderma, characterized by enhanced O-glycosylation, secreted much more proteins when relieved of the cell wall barrier (Perlińska-Lenart et al., 2006b). These results suggest that to obtain increased protein production and secretion both an enhancement of posttranslational modifications and weakening of the cell wall structure are required.

A Hansenula polymorpha strain carrying disruption of the pmt gene produced and secreted high amounts of normally $\mathrm{N}$-glycosylated proteins such as heterologously expressed human urinary type plasminogen activator, although in a non-glycosylated form (Agaphonov et al., 2005). The strain showed temperature sensitivity which was alleviated on osmotically ( $1 \mathrm{M}$ sorbitol) supported medium, indicating a cell wall integrity defect. In this strain stimulation of protein production and secretion concerned only N-glycoproteins, whereas both the production and secretion, and the affinity to chitin of under-Oglycosylated chitinase were significantly decreased.

Those results pointed at different roles of Oand $\mathrm{N}$-glycosylation for protein production, secretion, stability, localization and functioning. In yeast the integral plasma membrane proteins Ax12/Bud10 and Fus4 need Pmt4 O-mannosyltransferase for their O-mannosylation. In the pmt4 $\Delta$ mutant the Axl2/ Bud10 protein remained un-O-glycosylated and was probably recognized as improperly folded and became degraded in the Golgi apparatus (Sanders et al., 1999). Un-O-glycosylated Fus1p was accumulated in the late Golgi structures suggesting that O-glycosylation functions as a sorting determinant for cell surface delivery of Fus1p (Proszynski et al., 2004).

Disruption of the pmt1 gene in Trichoderma resulted in significant changes in the structure of the cell wall and the mutant was not able to grow without an osmotic stabilizer (1 M sorbitol) (GórkaNieć et al., 2008). This defect was not repaired by switching on the cell wall integrity pathway in contrast to what was observed in an Aspergillus pmtA disruptant, in which the amount of chitin in the cell wall was elevated (Oka et al., 2004; 2005).

Secretion of proteins in the Trichoderma pmt1 mutant was decreased while their glycosylation was not altered. Since Pmt proteins had been reported to be substrate-specific, one could conclude that those secretory proteins were not substrates of the PMTI protein $\mathrm{O}$-mannosyltransferase.

An attempt to stimulate the activity of protein $O$-mannosyltransferases by integration of additional copies of the pmt1 gene in Trichoderma was unsuccessful and resulted in silenced expression of the pmt1 gene and of some other pmts present in this fungus (Górka-Nieć et al., manuscript in preparation). The Trichoderma strain carrying additional copies of pmt1 secreted under-O- and N-glycosylated proteins, but in normal amounts. Since the cell wall of the pmt1-silenced mutant was weakened, but to a lesser extent than in the pmt1 disruptant, the strain could grow without $1 \mathrm{M}$ sorbitol. The possibility of cultivation of this strain without sorbitol eliminated an additional factor (1 M sorbitol) which influenced protein glycosylation and secretion in the pmt1 disruptant. A comparison of the Trichoderma pmt1-silenced strain with the $H$. polymorpha or $A$. awamori pmt1 disruptants supported our thesis that changes in protein glycosylation ability may differently influence protein production, glycosylation and secretion in different fungal species.

\section{CONCLUSIONS}

Generally, defects in protein O-glycosylation induce compensatory changes in cells that allow their growth. These changes include: activation of cell wall compensatory mechanisms, up-regulation of stress response, decrease of transcription of genes coding for glycosylated secretory proteins, and interactions between $\mathrm{O}-$ and N-glycosylation. The secreted proteins are under glycosylated and show an altered activity compared to the native ones.

On the other hand, enhanced O-glycosylation does not give effects opposite to those caused by decreased O-glycosylation. The same compensatory mechanisms could be switched on. Moreover, the higher activity of the O-glycosylation pathway could result in over-glycosylation and a changed biological activity of the secreted proteins. Nevertheless, O-glycosylation appears a promising site of manipulation to influence processes interesting from the biotechnological point of view. Effective processing of proteins in the endoplasmic reticulum and Golgi apparatus and their quicker relocation along the secretory structures allow a higher throughput. If, in addition, the cell wall of the fungus is permeable enough not to hamper the secretion itself, a higher production of secretory proteins may be achieved.

\section{Acknowledgements}

Work partially financed by the Committee for Scientific Research (KBN, Poland) grants 6P04B00621 and 1307/P01/2006/31 to J.S. Kruszewska.

\section{REFERENCES}

Adair WLJ, Cafmeyer N (1987) Characterization of the Saccharomyces cerevisiae cis-prenyltransferase required for 
dolichyl phosphate biosynthesis. Arch Biochem Biophys 259: 589-596.

Agaphonov MO, Packeiser AN, Chechenova MB, Choi E, Ter-Avanesyan MD (2001) Mutation of the homologue of GDP-mannose pyrophosphorylase alters cell wall structure, protein glycosylation and secretion in Hansenula polymorpha. Yeast 18: 391-402.

Agaphonov MO, Sokolov S, Romanova NV, Sohn J, Kim SY, Kalebina TS, Choi ES, Ter-Avanesyan MD (2005) Mutation of the protein-O-mannosyltransferase enhances secretion of the human urokinase-type plasminogen activator in Hansenula polymorpha. Yeast 22: 1037-1047.

Al-Sheikh H, Watson AJ, Lacey GA, Punt PJ, MacKenzie DA, Jeenes DJ, Pakula T, Penttila M, Alcocer MJ, Archer DB (2004) Endoplasmic reticulum stress leads to the selective transcriptional downregulation of the glucoamylase gene in Aspergillus niger. Mol Microbiol 53: 1731-1742.

Ashida H, Maeda Y, Kinoshita T (2006) DPM1, the catalytic subunit of dolichol-phosphate mannose synthase, is tethered to and stabilized on the endoplasmic reticulum membrane by DPM3. J Biol Chem 281: 896-904.

Beauvais A, Maubon D, Park S, Morelle W, Tanguy M, Huerre M, Perlin DS, Latge JP (2005) Two alpha(1-3) glucan synthases with different functions in Aspergillus fumigatus. Appl Environ Microbiol 71: 1531-1538.

Belgareh-Touze N, Corral-Debrinski M, Launhardt H, Galan JM, Munder T, Le Panse S, Haguenauer-Tsapis R (2003) Yeast functional analysis: identification of two essential genes involved in ER to Golgi trafficking Traffic 4: 607-617.

Chavan M, Suzuki T, Rekowicz M, Lennarz W (2003) Genetic, biochemical, and morphological evidence for the involvement of N-glycosylation in biosynthesis of the cell wall $\beta 1,6$-glucan of Saccharomyces cerevisiae. Proc Natl Acad Sci USA 100: 15381-15386.

Choi BK, Davidson RC, Hamilton SR, Kung DH, Li H, Miele RG, Nett JH, Wildt S, Gerngross TU (2003) Use of combinatorial genetic libraries to humanize N-linked glycosylation in the yeast Pichia pastoris. Proc Natl Acad Sci USA 100: 5022-5027.

Colussi PA, Taron CH, Mack JC, Orlean P (1997) Human and Saccharomyces cerevisiae dolichol phosphate mannose synthases represent two classes of the enzyme, but both function in Schizosaccharomyces pombe. Proc Natl Acad Sci USA 94: 7873-7878.

Conesa A, Punt PJ, van Luijk N, van den Hondel CAMJJ (2001) The secretion pathway in filamentous fungi: A biotechnological view. Fungal Gen Biol 33: 155-171.

Daleo GR, Hopp HE, Romero PA, Lezica R (1977) Biosynthesis of dolichol phosphate by subcellular fractions from liver. FEBS Lett 81: 411-414.

Davydenko SG, Juselius JK, Munder T, Bogengruber E, Jantti J, Keranen S (2004) Screening for novel essential genes of Saccharomyces cerevisiae involved in protein secretion. Yeast 21: 463-471.

De Bruyn A, Maras M, Schraml J, Herdewijn P, Contreras R (1997) NMR evidence for a novel asparagine-linked oligosaccharide on cellobiohydrolase I from Trichoderma reesei RUTC 30. FEBS Lett 405: 111-113.

Durand H, Clanet M, Tiraby G (1988) Genetic improvement of Trichoderma reesei for large scale cellulase production. Enzyme Microbiol Technol 10: 341-345.

Ecker M, Mrsa V, Hagen I, Deutzmann R, Strahl S, Tanner W (2003) O-mannosylation precedes and potentially controls the N-glycosylation of a yeast cell wall glycoprotein. EMBO reports 4: 628-632.

Ellgaard L, Helenius A (2003) Quality control in the endoplasmic reticulum. Mol Cell Biol 4: 181-191.
Fagerstam LG, Pettersson LG, Engström JA (1984) The primary structure of a $\beta$-1,4-glucan cellobiohydrolase from the fungus Trichoderma reesei QM9414. FEBS Lett 167: 309-315.

Gamauf C, Marchetti M, Kallio J, Puranen T, Vehmaanperä J, Allmaier G, Kubicek CP, Seiboth B (2007) Characterization of the bga1-encoded glucoside hydrolase family $35 \beta$-galactosidase of Hypocrea jecorina with galacto- $\beta$ D-galactanase activity. FEBS J 274: 1691-1700.

Garcia R, Cremata JA, Quintero O, Montesino R, Benkestock K, Stahlberg J (2001) Characterization of protein glycoforms with N-linked neutral and phosphorylated oligosaccharides: studies on the glycosylation of endoglucanase 1 (Cel7B) from Trichoderma reesei. Biotechnol Appl Biochem 33: 141-152.

Gentzsch M, Tanner W (1996) The PMT gene family: protein O-glycosylation in Saccharomyces cerevisiae is vital. EMBO J 15: 5752-5759.

Gentzsch M, Tanner W (1997) Protein-O-glycosylation in yeast: protein-specific mannosyltransferases. Glycobiology 7: 481-486.

Girrbach V, Strahl S (2003) Members of the evolutionarily conserved PMT family of protein O-mannosyltransferases form distinct protein complexes among themselves. J Biol Chem 278: 12554-12562.

Goto M (2007) Protein O-glycosylation in fungi. Diverse structure and multiple functions. Biosci Biotechnol Biochem 71: 1415-1427.

Goto M, Tsukamoto M, Kwon I, Ekino K, Furukawa K (1999) Functional analysis of O-linked oligosaccharides in threonine/serine-rich region of Aspergillus glucoamylase by expression in mannosyltransferase disruptants of yeast. Eur J Biochem 260: 596-602.

Górka-Nieć W, Bańkowska R, Palamarczyk G, Kruszewska JS (2007) Protein glycosylation in pmt mutants of Saccharomyces cerevisiae. Influence of heterologously expressed cellobiohydrolase II of Trichoderma reesei and elevated levels of GDP-mannose and cis-prenyltransferase activity. Biochim Biophys Acta 1770: 774-780.

Górka-Nieć W, Pniewski M, Kania A, Perlińska-Lenart U, Palamarczyk G, Kruszewska JS (2008) Disruption of Trichoderma reesei gene encoding protein O-mannosyltransferase I results in a decrease of the enzyme activity and alteration of cell wall composition. Acta Biochim Polon 55: 251-259.

Guillemette T, van Peij NNME, Goosen T, Lanthaler K, Robson GD, van den Hondel CAMJJ, Stam H, Archer DB (2007) Genomic analysis of the secretion stress response in the enzyme-producing cell factory Aspergillus niger. BMC Genomics 8: 158.

Harrison MJ, Nouwens AS, Jardine DR, Zachara NE, Gooley AA, Nevalainen H, Packer NH (1998) Modified glycosylation of cellobiohydrolase I from a high cellulase-producing mutant strain of Trichoderma reesei. Eur J Biochem 256: 119-127.

Harrison MJ, Wathugala IM, Tenkanen M, Packer NH, Nevalainen KMH (2002) Glycosylation of acetylxylan esterase from Trichoderma reesei. Glycobiology 12: 291-298.

Harty C, Strahl S, Romisch K (2001) O-mannosylation protects mutant alpha-factor precursor from endoplasmic reticulum-associated degradation. Mol Biol Cell 12: 1093-1101.

Helenius A, Aebi M (2001) Intracellular functions of Nlinked glycans. Science 291: 2364-2369.

Hollister J, Grabenhorst E, Nimtz M, Conradt H, Jarvis DL (2002) Engineering the protein N-glycosylation pathway in insect cells for production of biantennary, complex N-glycans. Biochemistry 41: 15093-15104. 
$\mathrm{Hu}$ JP, Lanthier P, White TC, McHugh SG, Yaguchi M, Thibault P (2001) Characterization of cellobiohydrolase I (Cel7A) glycoforms from extracts of Trichoderma reesei using capillary isoelectric focusing and electrospray mass spectrometry. J Chromatogr B Biomed Sci Appl 752: 349-68.

Hui JP, White TC, Thibault P (2002) Identification of glycan structure and glycosylation sites in cellobiohydrolase II and endoglucanases I and II from Trichoderma reesei. Glycobiology 12: 837-849.

Jenkins N, Marphy L, Thyler R (2008) Post-translational modifications of recombinant proteins: Significance for biopharmaceuticals. Mol Biotechnol 39: 113-118.

Jeoh T, Michener W, Himmel ME, Decker SR, Adney WS (2008) Implications of cellobiohydrolase glycosylation for use in biomass conversion. Biotechnol Biofuels 1: 10.

Klarskov K, Piens K, Ståhlberg J, Hoi PB, van Beeumen J, Claeyssens M (1997) Cellobiohydrolase I from Trichoderma reesei: identification of an active-site nucleophile and additional information on sequence including glycosylation pattern of the core protein. Carbohyd Res 304: 143-153.

Klis MF, Boorsma A, De Groot PWJ (2006) Cell wall construction in Saccharomyces cerevisiae. Yeast 23: 185-202.

Ko K, Ahn MH, Song M, Choo YK, Kim HS, Ko K, Joung $\mathrm{H}$ (2008) Glyco-engineering of biotherapeutic proteins in plants. Moll Cells 25: 494-503.

Kollar R, Reinhold BB, Petrakova E, Yeh HJC, Ashwell G, Drgonova J, Kapteyn JC, Klis FM, Cabib E (1997) Architecture of the yeast cell wall. J Biol Chem 272: 17762 17775.

Kruszewska J, Messner R, Kubicek CP, Palamarczyk G (1989) O-glycosylation of proteins by membrane fraction of Trichoderma reesei QM9414. J Gen Microbiol 135: 301-307.

Kruszewska J, Palamarczyk G, Kubicek CP (1990) Stimulation of exoprotein secretion by choline and Tween 80 in Trichoderma reesei QM 9414 correlates with increased activity of dolichol phosphate mannose synthase. J Gen Microbiol 136: 1293-1298.

Kruszewska J, Butterweck AH, Kurzątkowski W, Migdalski A, Kubicek CP, Palamarczyk G (1999) Overexpression of the Saccharomyces cerevisiae mannosylphosphodolichol synthase - encoding gene in Trichoderma reesei results in an increased level of protein secretion and abnormal cell ultrastructure. Appl Environm Microbiol 65: 2382-2387.

Kruszewska JS, Saloheimo M, Migdalski A, Orlean P, Penttilä M, Palamarczyk G (2000) Dolichol phosphate mannose synthase from the filamentous fungus Trichoderma reesei belongs to the human and Schizosaccharomyces pombe class of the enzyme. Glycobiology 10: 983-991.

Kubicek CP, Panda T, Schreferl-Kunar G, Messner R, Gruber F (1987) O-linked - but not N-linked - glycosylation is necessary for secretion of endoglucanase I and II by Trichoderma reesei. Can I Microbiol 33: 698-703.

Lennarz WJ (2007) Studies on oligosaccharyl transferase in yeast. Acta Biochim Polon 54: 673-677.

Maeda Y, Tomita S, Watanabe R, Ohishi K, Kinoshita T (1998) DPM2 regulates biosynthesis of dolichol phosphate-mannose in mammalian cells: correct subcellular localization and stabilization of DPM1, and binding of dolichol phosphate. EMBO J 17: 4920-4929.

Maeda Y, Tanaka S, Hino J, Kangawa K, Kinoshita T (2000) Human dolichol-phosphate-mannose synthase consists of three subunits: DPM1, DPM2, and DPM3. EMBO J 19: $2475-2482$.

Maras M, de Bruyn A, Schraml J, Hersewijn P, Claeyssens M, Fiers W, Contreras R (1997) Structural characteriza- tion of N-linked oligosaccharides from cellobiohydrolase I secreted by filamentous fungus Trichoderma reesei RUTC 30. Eur J Biochem 245: 617-625.

Muller Ch, McIntyre M, Hansen K, Nielsen J (2002) Metabolic engineering of the morphology of Aspergillus oryzae by altering chitin synthesis. Appl Environ Microbiol 68: 1827-1836.

Nakatsukasa K, Okada S, Umebayashi K, Fukuda R, Nishikawa S, Endo T (2004) Roles of O-mannosylation of aberrant proteins in reduction of the load for endoplasmic reticulum chaperones in yeast. J Biol Chem 279: $49762-49772$.

Oka T, Hamaguchi T, Sameshima Y, Goto M, Furukawa K (2004) Molecular characterization of protein O-mannosylation and its involvement in cell-wall synthesis in Aspergillus nidulans. Microbiology 150: 1973-1982.

Oka T, Sameshima Y, Koga T, Kim H, Goto M, Furukawa K (2005) Protein O-mannosyltransferase A of Aspergillus awamori is involved in O-mannosylation of glucoamylase I. Microbiol 151: 3657-3667.

Pakula TM, Laxell M, Huuskonen A, Uusitalo J, Saloheimo M, Penttila M (2003) The effects of drugs inhibiting protein secretion in the filamentous fungus Trichoderma reesei. Evidence for downregulation of genes that encode secreted proteins in the stressed cells. J Biol Chem 278: 45011-45020.

Pakula TM, Uusitalo J, Saloheimo M, Salonen K, Aarts RJ, Penttila M (2000) Monitoring the kinetics of glycoprotein synthesis and secretion in the filamentous fungus Trichoderma reesei: cellobiohydrolase I (CBHI) as a model protein. Microbiology 146: 223-232.

Palamarczyk G, Maras M, Contreras R, Kruszewska J (1998) Protein secretion and glycosylation in Trichoderma. In Trichoderma and Glocladium. Kubicek CP, Harman GE, eds, vol 1, pp 121-138. Taylor and Francis Ltd, London, UK.

Parodi AJ (2000) Protein glucosylation and its role in protein folding. Annu Rev Biochem 69: 69-93.

Penttila ME, Andre L, Lehtovaara P, Bailey M, Teeri TT, Knowles JKC (1988) Efficient secretion of two fungal cellobiohydrolases by Saccharomyces cerevisiae. Gene 63: 103-112.

Perlińska-Lenart U, Kurzątkowski W, Janas P, Kopińska A, Palamarczyk G, Kruszewska JS (2005) Protein production and secretion in an Aspergillus nidulans mutant impaired in glycosylation. Acta Biochim Polon 52: 195-205.

Perlińska-Lenart U, Bańkowska R, Palamarczyk G, Kruszewska JS (2006a) Overexpression of the Saccharomyces cerevisiae RER2 gene in Trichoderma reesei affects dolichol dependent enzymes and protein glycosylation. Fungal Genet Biol 43: 422-429.

Perlińska-Lenart U, Orłowski J, Laudy AE, Zdebska E, Palamarczyk G, Kruszewska JS (2006b) Glycoprotein hypersecretion alters the cell wall in Trichoderma reesei strains expressing the Saccharomyces cerevisiae dolichylphosphate mannose synthase gene. Appl Environ Microbiol 72: 7778-7784.

Proszynski TJ, Simons K, Bagnat M (2004) O-Glycosylation as a sorting determinant for cell surface delivery in yeast. Mol Biol Cell 15: 1533-1543.

Qin Y, Wei X, Liu X, Wang T, Qu Y (2008) Purification and characterization of recombinant endoglucanase of Trichoderma reesei expressed in Saccharomyces cerevisiae with higher glycosylation and stability. Protein Expr Purif 58: 162-167.

Sanders S, Gentzsch M, Tanner W, Herskowitz I (1999) Oglycosylation of Axl1/Bud10p by Pmt4p is required for its stability, localization and function in daughter cells. J Cell Biol 145: 1177-1188. 
Sato M, Sato K, Nishikawa S, Hirata A, Kato J, Nakano A (1999) The yeast RER2 gene, identified by endoplasmic reticulum protein localization mutations, encodes cisprenyltansferase, a key enzyme in dolichol synthesis. Mol Cell Biol 19: 471-483.

Sato M, Fujisaki S, Sato K, Nishimura Y, Nakano A (2001) Yeast Saccharomyces cerevisiae has two cis-prenyltransferases with different properties and localizations. Implication for their distinct physiological roles in dolichol synthesis. Genes Cells 6: 495-506.

Savel'ev AN, Eneyskaya EV, Isaeva-Ivanova LS, Shabalin KA, Golubev AM, Neustroev KN (1997) The carbohydrate moiety of $\alpha$-galactosidase from Trichoderma reesei. Glycoconjugate J 14: 897-905

Schmidt M, Strenk ME, Boyer MP, Fritsch BJ (2005) Importance of cell wall mannoproteins for septum formation in Saccharomyces cerevisiae. Yeast 22: 715-723.

Seidl V, Huemer B, Seiboth B, Kubicek CP (2005) A complete survey of Trichoderma chitinases reveals three distinct subgroups of family 18 chitinases. FEBS $J$ 272: 5923-5939.

Shahinian S, Dijkgraaf GJ, Sdicu AM, Thomas DY, Jakob CA, Aebi M, Bussey H (1998) Involvement of protein $N$-glycosyl chain glucosylation and processing in the biosynthesis of cell wall $\beta-1,6$-glucan of Saccharomyces cerevisiae. Genetics 149: 843-856.

Shenk B, Rush JS, Waechter ChJ, Aeby M (2001) An alternative cis-isoprenyltransferase activity in yeast that produces polyisoprenols with chain lengths similar to mammalian dolichols. Glycobiology 11: 89-98.

Shoemaker S, Schweickart V, Ladner M, Gelfand D, Kwok K, Myambo K, Innis M (1983) Molecular cloning of exo-cellobiohydrolase I derived from Trichoderma reesei strain L27. Bio/Technology 1: 691-699.

Shridas P, Rush JS, Waechter ChJ (2003) Identification and characterization of a cDNA encoding a long-chain cis- isoprenyltransferase involved in dolichyl monophosphate biosynthesis in the ER of brain cells. Biochem Biophys Res Comm 312: 1349-1356.

Spiriti J, Bogani F, van der Vaart A, Ghirlanda G (2008) Modulation of protein stability by O-glycosylation in a designed Gc-MAF analog. Biophys Chem 134: 157-167.

Stals I, Sandra K, Devreese B, Van Beeumen J, Claeyssens M (2004) Factors influencing glycosylation of Trichoderma reesei cellulases. II: N-glycosylation of Cel7A core protein isolated from different strains. Glycobiology 14 : 725-735.

Szkopińska A, Karst F, Palamarczyk G (1996) Products of S. cerevisiae cis-prenyltransferase activity in vitro. Biochimie 78: 111-116.

Uccelletti D, Staneva D, Rufini S, Rufini S, Vencov P, Palleschi C (2005) Enhanced secretion of heterologous proteins in Kluyveromyces lactis by overexpression of the GDP-mannose pyrophosphorylase, KIPsa1p. FEMS Yeast Res 5: 735-746.

Wildt S, Gerngross TU (2005) The humanization of N-glycosylation pathways in yeast. Nature Rev Microbiol 3: 119-128.

Williamson G, Belshaw NJ, Williamson MP (1992) O-glycosylation in Aspergillus glucoamylase. Conformation and role in binding. Biochem J 282: 423-428.

Yan A, Lennarz WJ (2005) Unraveling the mechanism of protein N-glycosylation. J Biol Chem 280: 3121-3124.

Zakrzewska A, Palamarczyk G, Krotkiewski H, Zdebska E, Saloheimo M, Penttilä M, Kruszewska JS (2003) Overexpression of the gene encoding GTP-mannose-1-phosphate guanyltransferase, $m p g 1$, increases cellular GDPmannose levels and protein mannosylation in Trichoderma reesei. Appl Environ Microbiol 69: 4383-4389. 